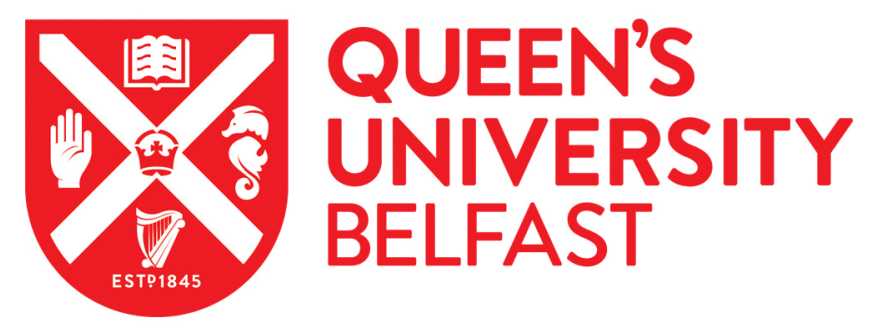

\title{
An interferon lambda 4-associated variant in the hepatitis C virus RNA polymerase affects viral replication in infected cells
}

Bamford, C. G. G., \& McLauchlan, J. (2021). An interferon lambda 4-associated variant in the hepatitis C virus RNA polymerase affects viral replication in infected cells. Journal of General Virology, 102(2).

https://doi.org/10.1099/jgv.0.001495

Published in:

Journal of General Virology

Document Version:

Publisher's PDF, also known as Version of record

Queen's University Belfast - Research Portal:

Link to publication record in Queen's University Belfast Research Portal

Publisher rights

(c) 2021 The Authors.

This is an open-access article distributed under the terms of the Creative Commons Attribution NonCommercial Licence.

\section{General rights}

Copyright for the publications made accessible via the Queen's University Belfast Research Portal is retained by the author(s) and / or other copyright owners and it is a condition of accessing these publications that users recognise and abide by the legal requirements associated with these rights.

Take down policy

The Research Portal is Queen's institutional repository that provides access to Queen's research output. Every effort has been made to ensure that content in the Research Portal does not infringe any person's rights, or applicable UK laws. If you discover content in the Research Portal that you believe breaches copyright or violates any law, please contact openaccess@qub.ac.uk. 


\title{
An interferon lambda 4-associated variant in the hepatitis $C$ virus RNA polymerase affects viral replication in infected cells
}

\author{
Connor G.G. Bamford ${ }^{1,2}$ and John McLauchlan ${ }^{1, *}$
}

\begin{abstract}
Host IFNL4 haplotype status contributes to the development of chronic hepatitis C virus (HCV) infection in individuals who are acutely infected with the virus. In silico studies revealed that specific amino acid variants at multiple sites on the HCV polyprotein correlate with functional single-nucleotide polymorphisms (SNPs) in the IFNL4 locus. Thus, SNPS at the IFNL4 locus may select variants that influence virus replication and thereby the outcome of infection. Here, we examine the most significantly IFNL4-associated amino acid variants that lie in the 'lambda (L) 2 loop' of the HCV NS5B RNA polymerase. L2 loop variants were introduced into both sub-genomic replicon and full-length infectious clones of HCV and viral replication was examined in the presence and absence of exogenous IFN $\lambda 4$. Our data demonstrate that while mutation of the NS5B L2 loop affects replication, individual IFNL4-associated variants have modest but consistent effects on replication in both the presence and absence of IFN $\lambda$ 4. Given the strong genetic association between these variants and IFNL4, these data suggest a nuanced effect of each individual position on viral replication, the combined effect of which might mediate resistance to the effects of IFN $\lambda 4$.
\end{abstract}

Clearance of hepatitis $\mathrm{C}$ virus (HCV) is associated with genetic and functional variation in the human IFN lambda 4 (IFNL4) gene [1]. Recent analyses of unbiased 'genome-togenome' variant association has also identified correlations between HCV genetic polymorphisms at specific sites across the virus genome and IFNL4 variation [2-4]. This suggests that virus populations in those producing functional IFNL4 differ from those generating the non-functional or less potent forms of the protein. Thus, there may be an interaction between host and viral genetic variants that ultimately affects viral chronicity. A previous report characterized one variant in NS5A, which was linked to serum viral load in individuals expressing functional IFNL4, indicating that this IFNL4-associated site may affect virus replication in the sub-genomic replicon (SGR) system [2]. However, studies on robust full-length $\mathrm{HCV}$ cell culture (HCVcc) infectious systems have not been carried out. Furthermore, the most significant IFNL4-associated variant (A150V) in the NS5B protein from studies on $\mathrm{HCV}$ gt3a sequences has not been examined in detail for any contribution to the viral replication process in such systems [2-4].
The region encompassing A150V and an additional cluster of IFNL4-associated variants is located between amino acids (aa) aa2567 to aa2576 in the HCV polyprotein and lies towards the N-terminus of the virus-encoded NS5B RNAdependent RNA polymerase (RdRp; positions 2567, 2568, 2570 and 2576 correspond to residues 147, 148, 150 and 156 in NS5B; Fig. 1a). This region corresponds to a relatively variable segment termed 'motif $\mathrm{F}$ ' in the N-terminal 'fingers' domain of conserved viral RdRp enzymes of RNA viruses, and has been termed the lambda (L) 2 loop (Fig. 1a, b) $[5,6]$. Comparison of HCV RdRp sequences across other families in the Flaviviridae, including the flaviviruses, pegiviruses and pestiviruses, revealed invariant amino acid residues flanking the L2 loop in regions termed F1 and F2. Upstream of the L2 loop is a conserved KXE motif ( $\mathrm{X}=\mathrm{N} / \mathrm{K} / \mathrm{R}$ depending on the virus family), while in the downstream region are invariant lysine, arginine and isoleucine residues in a KXXRXI motif (Fig. 1b). Within the L2 loop there were no invariant residues. Sequence comparison of amino acid sequences for the L2 loop among and within HCV genotypes further demonstrated the variability of this region (Fig. 1c). Positions 148 and 150 showed the greatest variability, although we also noted the lack

Received 19 June 2020; Accepted 18 August 2020; Published 08 September 2020

Author affiliations: 'MRC-University of Glasgow Centre for Virus Research, 464 Bearsden Rd, Glasgow, G61 1QH, Scotland, UK; ${ }^{2}$ Wellcome-Wolfson Institute for Experimental Medicine, Queen's University of Belfast, 97 Lisburn Rd, Belfast, BT97BL, Northern Ireland, UK.

*Correspondence: John McLauchlan, john.mclauchlan@glasgow.ac.uk

Keywords: hepatitis C virus; HCV; IFN入4; NS5B; RNA replication.

Abbreviations: CM, conditioned media; Gluc, gaussian luciferase; gt, genotype; HCV, hepatitis C virus; HCVcc, HCV cell culture; IFNL4, interferon

lambda 4; IVT, in vitro transcription; m.o.i., multiplicity of infection; SNP(s), single nucleotide polymorphism(s); TCID50, median tissue culture

infectious dose; WT, wild-type.

$001495 \odot 2021$ The Authors

(c) (1) (8) This is an open-access article distributed under the terms of the Creative Commons Attribution NonCommercial License. 
a

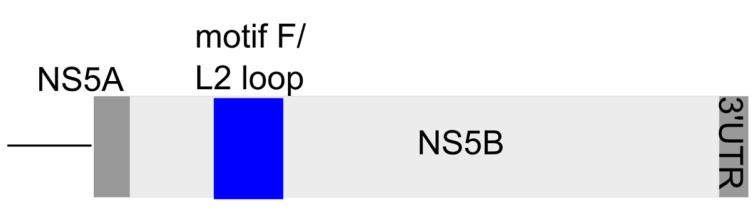

b

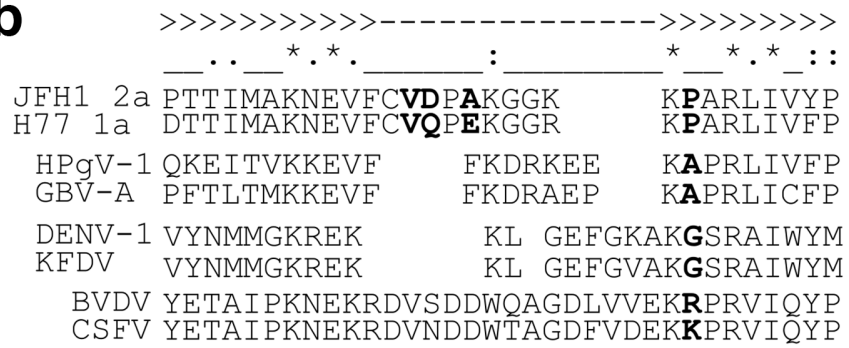

C

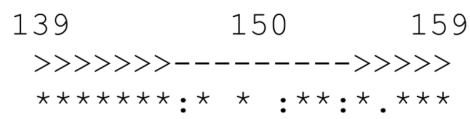

1 a MAKNEVECVQ̄ $\overline{\mathbf{E}}$ KGGRKPARL

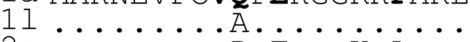

$2 a \ldots \ldots \ldots$ D. T.........

$2 a \ldots \ldots \ldots$ D.A..K....

$2 a \ldots \ldots$....IR.K.A.

3a.................

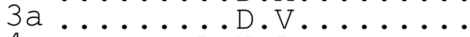

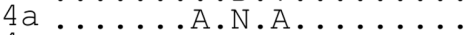

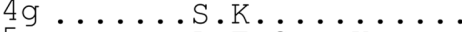

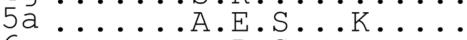

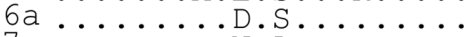

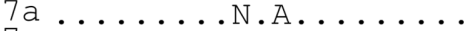

$7 \mathrm{c} \ldots . . . \mathrm{N} . \mathrm{L} \ldots \mathrm{A}$....

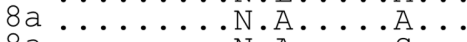

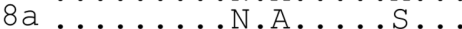

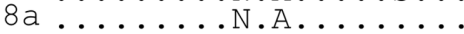

d

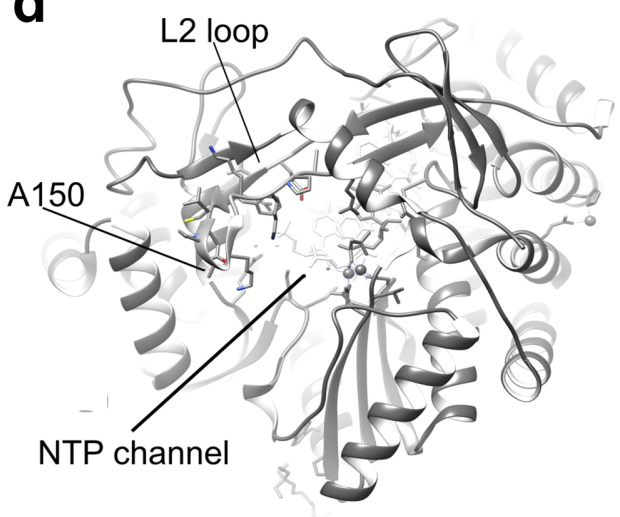

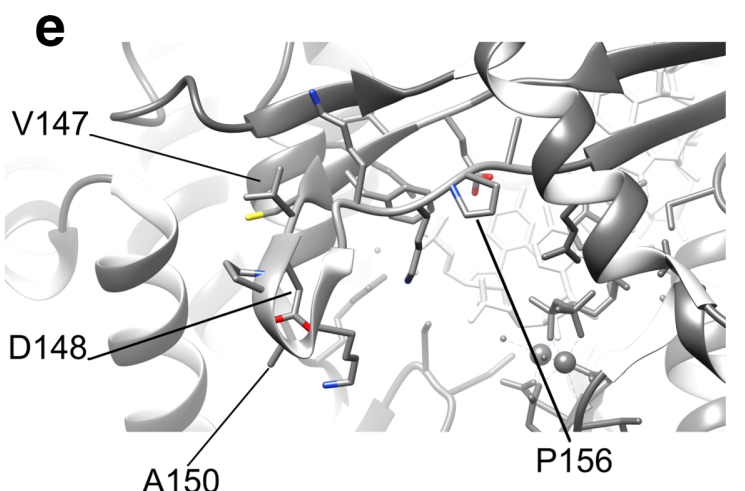

Fig. 1. Location of IFNL4-associated positions in the L2 loop of HCV NS5B RNA polymerase. (a) Schematic of the 3' end of the HCV genome showing the location of the NS5B motif F/L2 loop. (b, c) Amino acid alignment of the region encompassing motif $F$ in the Flaviviridae (b) and in HCV reference sequences (selected to highlight sequence diversity) for genotypes 1-8 (c). IFNL4-associated positions are highlighted in bold in HCV reference sequences [for gt1a and gt2a in (b) and gt1a in (c)]. Invariant $\left(^{*}\right)$, conserved (:) and partially conserved (.) positions are highlighted directly above the alignment. Secondary structural elements in the HCV NS5B RNA polymerase are indicated above sequences (>> for $\beta$-strands for F1 and F2; -- for the non-structured L2 loop). HCV gt2a JFH1: AB047639.1; HCV gt1a H77: AF009606.1; human pegivirus 1: BAA19580.1; simian pegivirus/GB-virus A: AHH32939.1; dengue virus serotype 1 (DENV-1): QFS19562.1; Kyasanur Forest disease virus (KFDV): AXB87737.1; bovine viral diarrhoeal virus (BVDV): AAF82566.1; classical swine fever virus (CSFV): AYE19937.1. HCV subtype accession numbers are as follows: 1a_AF009606, 1 I_KC248193, 2a_D00944, 2a_AB047639, 2a_HQ639944, 3a_D17763, 3a_JN714194, 4a_Y11604, 4g_FJ462432, 5a_AF064490, 6a_Y12083, 7a_EF108306, 7_KU861171.1, 8_pt1 MH590698.1, 8_pt2 MH590699.1 and 8_pt4 MH590701.1. (d, e) Structure of the L2 loop region in the HCV NS5B RNA polymerase. IFNL4associated positions are shown with amino acid side-chains on the 4wti pdb structure of HCV gt2a JFH1 NS5B. The L2 loop is shown relative to the NTP channel and residue A150 (d) and IFNL4-associated positions are indicated in a higher magnification image (e).

of sequence identity at positions 146 and 154 . In addition, the proline residue in the $\mathrm{F} 2$ region displayed some intergenotype diversity. Based on the crystal structure of HCV NS5B, the L2 loop corresponds to a flexible surface-exposed loop in the closed conformation of the protein where it extends outwards and over the nucleotide tunnel (Fig. 1d, e) [6]. Amino acids at positions 148 and 150 with the greatest variability lie at the extremity of the loop.

Based on the above analyses, we constructed four SGR and HCVcc L2 loop mutants in a JFH1 gt2a SGR construct containing a GLuc reporter and the Jc1 HCVcc infectious clone $[7,8]$. We chose to utilize the JFH1/Jc1 system because the documented IFNL4-associated variable position 150 is found not only in gt 3 a but also in gt 2 sequences. Therefore we were able to exploit the high replication capability of the wellcharacterized JFH1/Jc1 system. Our strategy was to create substitutions at positions 148 (D148A), 150 (A150V) and a double substitution at positions 148 and 150 (D148Q.A150E), thereby reconstituting the gtla sequences at these positions (Fig. 1c). Gt1a is considered to be a relatively IFN-resistant 
HCV subtype and these positions have been implicated in this phenotype as well as being associated with the IFNL4/IL28B genotype [9]. We also constructed a P156A variant, identified as being associated with the IFNL4 genotype in four HCV subtypes [2-4] to determine whether altering this position in the $\mathrm{F} 2$ region created a functional defect that would affect replication. Briefly the NS5B-coding region was sub-cloned into a plasmid vector and site-directed mutagenesis was used to alter residues before transferring the mutated fragments into the SGR plasmid and then into HCVcc plasmids. The mutagenesis primers used are available on request. All mutations in the final constructs were confirmed by sequencing.

RNA from the SGR constructs was generated by in vitro transcription (IVT) using the manufacturer's instructions (T7 RiboMAX, Promega, UK) and transfected (200 ng) into subconfluent monolayers of Huh7 cells in 96-well plates using Lipofectamine 2000 ( $\left.1 \mu \mu^{-1} \mathrm{~g}^{-1} \mathrm{RNA}\right)$ using the manufacturer's instructions (Thermo Fisher Scientific, UK). GLuc activity in $5 \%$ of the supernatant $(10 \mu \mathrm{l})$ was assessed at $4,24,48$ and $72 \mathrm{~h}$ post-transfection using the manufacturer's instructions. The reporter activity for each construct was compared to that for a replication-defective 'GND' mutant (Fig. 2a). Each of the mutant constructs plateaued around $48 \mathrm{~h}$ post-transfection and reached peak activity by $72 \mathrm{~h}$, achieving levels that were not significantly different from those of the wild-type (WT) construct. However, we noted that D148A gave 10-fold lower luciferase activity at 24 and $48 \mathrm{~h}$ post-transfection. Both the double mutant D148Q.A150E and A150V constructs also yielded less activity than the WT ( fivefold and $\sim$ twofold, respectively) at earlier times post-transfection.

The same four mutants were introduced into the Jc1 HCVcc system to allow analysis of viral multicycle replication. Following IVT of HCVcc RNA and electroporation into Huh7 cells, virus-containing supernatants were harvested at least 3 days later and infectivity was titrated by $\mathrm{TCID}_{50}$ using an NS5A-specific antibody [10]. All RNAs produced infectious virus, although D148A yielded $\sim 100$-fold fewer infectious progeny (Fig. 2b). Sequences of the mutated versions of HCVcc were confirmed by Sanger sequencing of RT-PCR amplicons; no changes at the consensus level were found apart from D148A, which failed to yield an RT-PCR product, presumably due to reduced replication of viral RNA (data not shown). Assessment of HCVcc RNA accumulation at $72 \mathrm{~h}$ post-infection following infection of Huh7 cells infected at a multiplicity of infection (m.o.i.) of 0.01 showed that while HCVcc viral RNA was detected by RT-qPCR as described previously [7] in all infections, only D148A and D148Q. A150E showed major differences (Fig. 2b). D148A gave a 100 -fold reduction in RNA accumulation, consistent with the lower yield of virus following transfection of IVT viral RNA, and D148Q.A150E a more modest 2-fold reduction in RNA accumulation. A150V and P156A led to slight (less than 1.5 -fold) but consistent increases in viral RNA accumulation.

One of our main objectives in the study was to examine whether A150V, which is associated with host IFNL4 genotype, would affect multicycle HCV replication in the presence or absence of IFN $\lambda 4$ using an in vitro model system. We have previously established a system to test the antiviral activity of WT human IFN $\lambda 4$ in cases of HCV infection using conditioned media (CM) that contained IFN $\lambda 4$ secreted by cells transfected with a plasmid expressing the protein [11]. Using the same approach, our prior study also examined the impact of IFN $\lambda 3$ on infection, which demonstrated that it had a far greater antiviral effect. In the following experiments, we utilized CM containing IFN $\lambda 3$ to determine the maximal impact of IFN $\lambda$ proteins on HCV RNA replication and infection, but not as a comparison between IFN $\lambda 3$ and IFN $\lambda 4$ in these systems. Moreover, to determine the impact of IFN $\lambda 4$ on viral RNA replication and virus infection, we utilized maximal amounts of CM containing IFN $\lambda 4$ that would not induce toxic effects.

Huh7 cells, treated with exogenous transfected-cell CM containing IFNs, were transfected with WT and mutant SGR RNAs or infected with WT and mutant HCVcc; GLuc activity was assayed at $48 \mathrm{~h}$ post-transfection (for SGR) and viral RNA measured at $72 \mathrm{~h}$ post-infection (for $\mathrm{HCVcc}$ ). Given the low replication of SGR and HCVcc constructs carrying the D148A mutation, we excluded these constructs from further analysis. As expected, pre-treatment of Huh7 cells with exogenous IFN $\lambda$ s gave a greater reduction with IFN $\lambda 3$ compared to IFN $\lambda 4$ for Gluc activity from SGR (Fig. 2c, d) and viral RNA from HCVcc constructs (Fig. 2) compared to cells treated with a control CM from EGFP plasmid-transfected controls. These results were similar to those in our previous analyses [11]. SGRs containing mutations had reduced replication in EGFP-CM-treated cells compared to WT (Fig. 2c), consistent with the data presented in Fig. $2 \mathrm{a}$ at $48 \mathrm{~h}$ post-transfection (Fig. 2a). IFN $\lambda$ pre-treatment inhibited SGR replication and, by normalizing the data to those from EGFP CM-treated cells, the mutations introduced into the SGR had a modest effect on replication in IFN $\lambda 4$-treated cells, with all mutants yielding approximately $\sim 1.5$-fold higher Gluc levels compared to the WT construct treated with IFN $\lambda 4$ (Fig. 2d). In the case of the P156A mutation, this higher level of replication in the presence of IFN $\lambda 4$ achieved statistical significance (Fig. 2d). Similar to the data in Fig. 2b, the HCVcc D148Q.A150E mutant had reduced replication in EGFP-CM-treated cells compared to WT (Fig. 2e). IFN $\lambda$ pre-treatment inhibited HCVcc RNA synthesis and, from normalization with EGFP CM-treated cells, showed that the D148Q.A150V mutant led to similar RNA accumulation to that of the WT construct (Fig. 2f). However, the A150V and P156A substitutions yielded a $\sim 1.5$-fold increase in RNA levels, similar to what was observed in SGR assays, achieving statistical significance in the case of A150V (Fig. 2f), suggestive of a consistently lower reduction in replication compared to the $\mathrm{WT}$ construct in the context of prior IFN $\lambda 4$ treatment. We conclude that in the presence of exogenous IFN $\lambda 4$, viral replication is modestly elevated for the A150V and P156A variants, located in the L2 loop in NS5B.

All of the variants introduced into the L2 loop and F2 region in the replicon and infectious systems were natural polymorphisms that occur in at least one HCV subtype (Fig. 1c). Since 

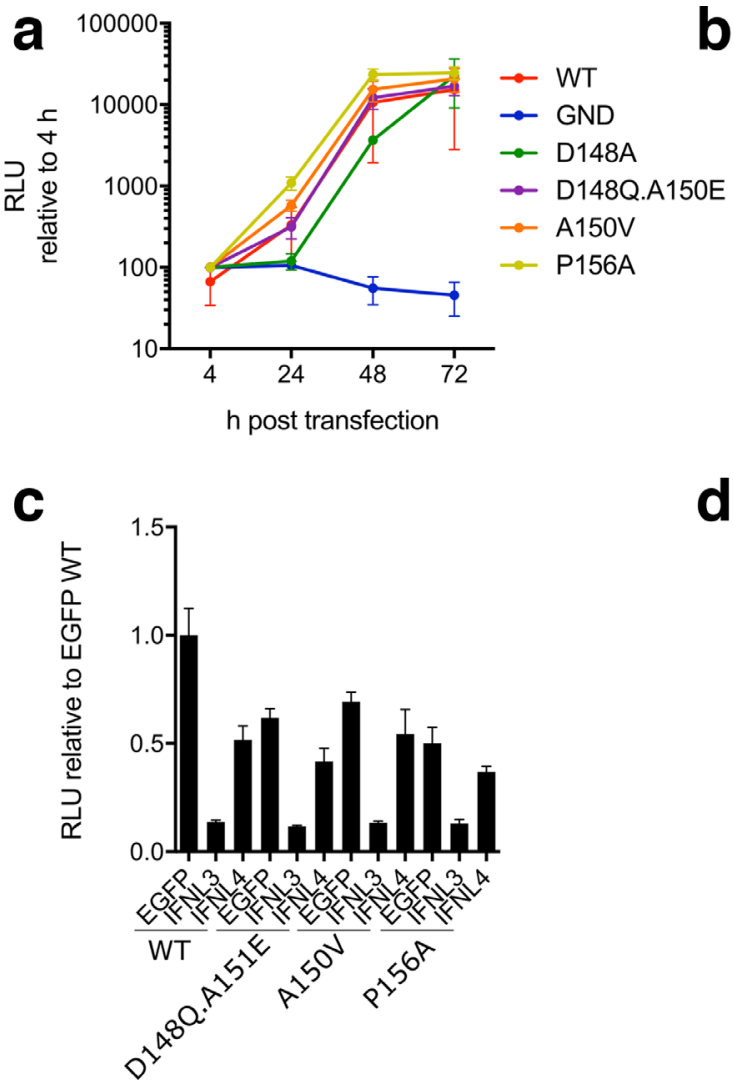

e

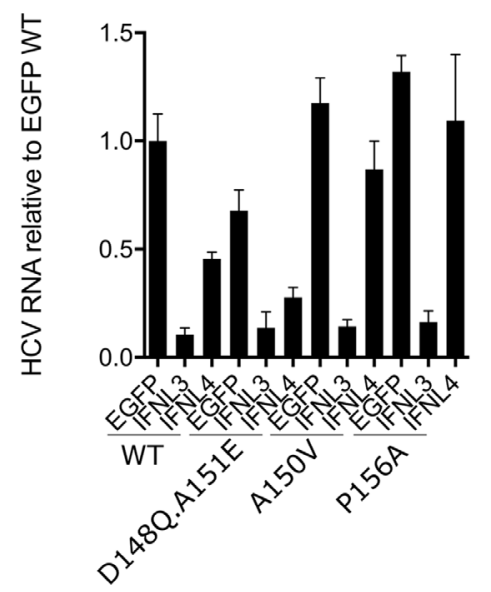

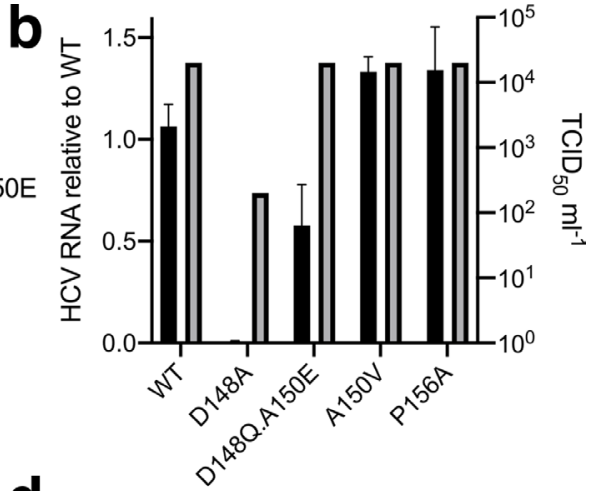

d

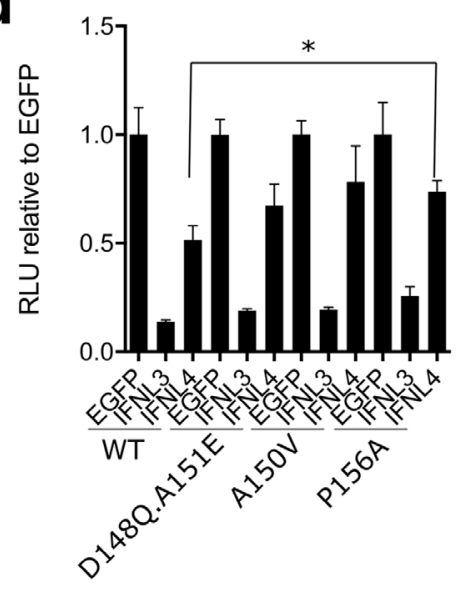

f

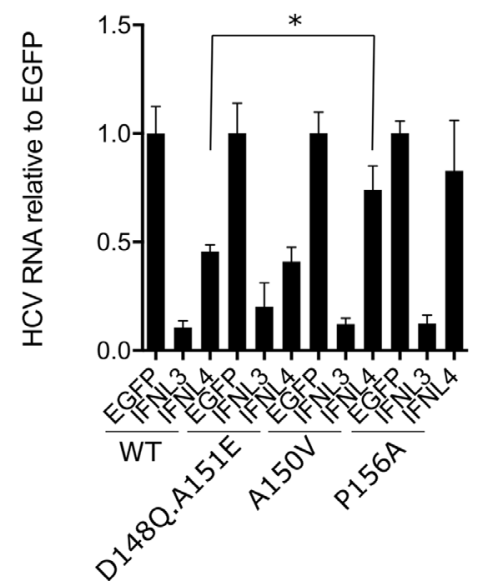

Fig. 2. Effect of IFNL4-associated variants in the NS5B L2 loop on viral RNA replication in the presence and absence of IFN $\lambda$ proteins. (a) Gluc activity at 4, 24, 48 and $72 \mathrm{~h}$ post-transfection of Huh7 cells with in vitro-transcribed SGR RNA. SGR constructs used are indicated and include a replication-incompetent GND control. Data are shown relative (\%) to the $4 \mathrm{~h}$ Gluc activity for each construct ( $n=5)$. (b) $\mathrm{TCID}_{50}$ values for HCVcc harvested at $72 \mathrm{~h}$ following electroporation of IVT viral RNA (shown in grey). The graph also shows viral genomic RNA abundance (shown in black) at $72 \mathrm{~h}$ post-infection following infection of Huh7 cells at a, m.o.i. of 0.01 with the indicated WT and mutant HCVcc viruses. Data for viral genomic RNA abundance are shown relative to WT HCVcc RNA, which were normalized to 1 ( $n=2$ ). (c-f) Sensitivity of WT and mutant SGRs (c, d) or HCVcc (e, f) to addition of exogenous IFN $\lambda 3$ and IFN 4 compared to an EGFP control. Gluc activity (c, d) or HCV genome RNA abundance (e, f) at $48 \mathrm{~h}$ post-transfection of Huh7 cells with in vitro-transcribed SGR RNA or $72 \mathrm{~h}$ post-infection following infection with HCVcc (m.o.i. of 0.01) following treatment (24h) with IFN $\lambda 3$ - or IFN $\lambda 4$ CM (1:4) as well as the EGFP-negative control CM. Data shown relative to WT SGR RNA/HCVcc from EGFP-treated cells (c, e) or to each construct EGFP-treated control (d, f) normalized to ' 1 ' ( $n=2$ ). Abundance of viral genomic RNA was measured at $72 \mathrm{~h}$ post-infection relative to GAPDH mRNA. All data show mean (+/- standard error or the mean). Statistical significance was measured using a two-tailed Student's $t$-test with * indicating $P=>0.05$. 
they were natural variants, we expected that they might have modest effects on viral RNA replication and virion production. However, we observed a substantial drop in viral RNA levels at earlier times in the replicon assay and poor yields of virus for the D148A substitution. An alanine residue is only found at position 148 for gtll, a rare subtype that is typically found in sub-Saharan Africa. By contrast, replacing both residues at positions 148 and 150 recreated the most frequently found amino acids in gtla (D148Q.A150E) and replication was decreased by $<$ twofold. Moreover, the other variants that were studied (A150V and P156A) had no overall effect on replication in the SGR system when compared to the WT construct and indeed gave a modest increase in viral RNA in the infectious model. Therefore, except for D148A, the substitutions were well tolerated in NS5B.

Recent molecular dynamics data have provided greater insight into the likely function of the HCV F domain in organizing the entry of nucleotides for access to the active site as well as the exit of pyrophosphate during the replication process [12]. Prior to nucleotide access to the active site, the F domain coordinates nucleotide reorientation and base stabilization through rearrangement of salt bridge interactions involving K151 (with either D352 or D387 depending on conformation) and R158 (with E143). The other residues comprising the F1L2-F2 region are likely to provide functional properties, such as coordinating the opening/closure of the nucleotide tunnel, structural flexibility and perhaps some nucleotide selectivity. Furthermore, a K151R mutation rescued infectivity of a P7 mutant HCVcc but had no measurable effect on replication or NS5B activity in vitro [13]. Another study identified a potential interaction between the L2 loop and domain II of NS5A [14]. Thus, the L2 loop may participate in interactions with other regions of NS5B (for RNA replication) and with other viral proteins such as NS5A and P7/NS2 (e.g. for assembly). Both D148 and A150 are located on the surface of L2, facing downwards towards incoming nucleotides. These residues lie immediately to the N-terminal side of residue K151, which is nearly completely invariant and forms a critical salt bridge with D352, bisecting the nucleotide entry site in the closed conformation of the L2 loop. The presence of variant amino acid residues immediately upstream of K151 could have modulatory effects on the behaviour of the L2 loop. Clearly, from our data, the D148A substitution has a substantial impact on viral replication, suggesting that it impairs the function of the L2 loop in the context of the HCVgt2a strain JFH1/Jc-1. The effect of D148A on replication in the SGR system was only apparent at early times post-transfection of subgenomic viral RNA and, by $72 \mathrm{~h}$, RNA replication was indistinguishable from that of the WT construct. The differences observed at $72 \mathrm{~h}$ between the SGR and infectious assays (compare Fig. 2a, b) may reflect the multi-cycle nature of infection with the JFH1/Jc-1 system. Alternatively, D148A could influence not only viral RNA replication, but also virion assembly. By contrast, both double variants at positions 148 and 150 (D148Q.A150E) and a single variant at position $150(\mathrm{~A} 150 \mathrm{~V})$ are well tolerated. Thus, it is possible to introduce intragenotypic and intergenotypic substitutions into L2 without disrupting function. This indicates a degree of redundancy in the L2 loop sequences. However, it is possible that certain variants have subtle effects on the selectivity of incoming nucleotides. For example, the A150V variant gave reduced susceptibility to the nucleotide analogue sofosbuvir, which is a clinically approved and highly potent direct-acting antiviral [15]. This reduced potency could arise from lower capacity of the A150V substitution to allow sofosbuvir entry into the catalytic site of the polymerase. Furthermore, we see differences between the HCV subgenomic replicon and infectious virus assays for A150V (and P156A), such that these mutations affect viral replication in the replicon but not the $\mathrm{HCVcc}$ system. This may suggest differing roles of these sites in replication compared to assembly.

Our data reveal that mutations A150V and P156A influence viral replication in the presence of exogenous IFN $\lambda 4$, such that, for both variants, the reduction in replication was lower in both SGR and HCVcc systems compared to the WT control. These results suggest that V150 and A156 confer slight resistance to IFN $\lambda 4$. The higher prevalence for V150 in those with IFNL4 alleles that produce functional protein would suggest a fitness advantage for this variant, which may be consistent with the partial IFN $\lambda 4$ resistance observed in our assays. However, viral load is lower in chronic infection in individuals who have IFNL4 alleles that produce IFN $\lambda 4$ compared to those who fail to make functional protein $[2,3]$. Thus, our data illustrate the challenge with aligning in vitro results to understand the mechanisms underlying in vivo findings. It is possible that there are epistatic effects, which would be difficult to ascertain with in vitro methods that do not recapitulate infection by natural strains. In conclusion, we have characterized the replicative effects of variants associated with the IFNL4 genotype that could serve as the basis for further studies on the role of viral variability in physiologically relevant in vitro models.

Funding information

This work was supported by the UK Medical Research Council (MC_UU_12014/1).

Acknowledgements

We wish to thank undergraduate students Maria Chalmers and Aqsa Sharif for their help with this project.

Author contributions

C. B. and J. M. conceived the project and established the goals of the study; C. B. conducted all experiments; C. B. and J. M. analysed and interpreted the data; C. B. and J. M. were responsible for writing and preparing the manuscript.

Conflicts of interest

The authors declare that there are no conflicts of interest.

References

1. Prokunina-Olsson L, Muchmore B, Tang W, Pfeiffer RM, Park H et al. A variant upstream of IFNL3 (IL28B) creating a new interferon gene IFNL4 is associated with impaired clearance of hepatitis C virus. Nat Genet 2013;45:164-171

2. Ansari MA, Pedergnana V, L C Ip C, Magri A, Von Delft A et al. Genome-to-genome analysis highlights the effect of the human innate and adaptive immune systems on the hepatitis $\mathrm{C}$ virus. Nat Genet 2017:49:666-673. 
3. Ansari MA, Aranday-Cortes E, Ip CLC, da Silva Filipe A, Lau SH et al. Interferon lambda 4 impacts the genetic diversity of hepatitis C virus. eLife 2019;8:e42463.

4. Chaturvedi N, Svarovskaia ES, Mo H, Osinusi AO, Brainard DM et al. Adaptation of hepatitis $C$ virus to interferon lambda polymorphism across multiple viral genotypes. eLife 2019;8:e42542.

5. Selisko B, Papageorgiou N, Ferron F, Canard B. Structural and functional basis of the fidelity of nucleotide selection by flavivirus RNA-dependent RNA polymerases. Viruses 2018;10:59.

6. Bressanelli S, Tomei L, Roussel A, Incitti I, Vitale RL et al. Crystal structure of the RNA-dependent RNA polymerase of hepatitis C virus. Proc Natl Acad Sci U S A 1999:96:13034-13039.

7. Domingues $P$, Bamford CGG, Boutell C, McLauchlan J. Inhibition of hepatitis $C$ virus RNA replication by ISG15 does not require its conjugation to protein substrates by the HERC5 E3 ligase. J Gen Virol 2015;96:3236-3242.

8. Pietschmann T, Kaul A, Koutsoudakis G, Shavinskaya A, Kallis S et al. Construction and characterization of infectious intragenotypic and intergenotypic hepatitis C virus chimeras. Proc Natl Acad Sci USA 2006;103:7408-7413.

9. Rose R, Markov PV, Lam TT, Pybus OG. Viral evolution explains the associations among hepatitis $\mathrm{C}$ virus genotype, clinical outcomes, and human genetic variation. Infect Genet Evol 2013;20:418-421.
10. Lindenbach BD, Meuleman P, Ploss A, Vanwolleghem $T$, Syder AJ et al. Cell culture-grown hepatitis $C$ virus is infectious in vivo and can be recultured in vitro. Proc Natl Acad Sci U S A 2006;103:3805-3809.

11. Bamford CGG, Aranday-Cortes E, Filipe IC, Sukumar S, Mair D et al. A polymorphic residue that attenuates the antiviral potential of interferon lambda 4 in hominid lineages. PLoS Pathog 2018;14:e1007307.

12. Ben Ouirane $K$, Boulard Y, Bressanelli S. The hepatitis $C$ virus RNAdependent RNA polymerase directs incoming nucleotides to its active site through magnesium-dependent dynamics within its $F$ motif. J Biol Chem 2019:294:7573-7587.

13. Gouklani H, Bull RA, Beyer C, Coulibaly F, Gowans EJ et al. Hepatitis $\mathrm{C}$ virus nonstructural protein $5 \mathrm{~B}$ is involved in virus morphogenesis. J Virol 2012;86:5080-5088.

14. Dujardin M, Cantrelle F-X, Lippens G, Hanoulle X. Interaction study between HCV NS5A-D2 and NS5B using 19F NMR. J Biomol NMR 2018;70:67-76.

15. Wing PAC, Jones $M$, Cheung $M$, DaSilva $S$, Bamford $C$ et al. Amino acid substitutions in genotype $3 \mathrm{~A}$ hepatitis $\mathrm{C}$ virus polymerase protein affect responses to sofosbuvir. Gastroenterology 2019;157:692-704.

\section{Five reasons to publish your next article with a Microbiology Society journal}

1. The Microbiology Society is a not-for-profit organization.

2. We offer fast and rigorous peer review - average time to first decision is $4-6$ weeks.

3. Our journals have a global readership with subscriptions held in research institutions around the world.

4. $80 \%$ of our authors rate our submission process as 'excellent' or 'very good'.

5. Your article will be published on an interactive journal platform with advanced metrics.

Find out more and submit your article at microbiologyresearch.org. 